\title{
Two novel compound heterozygous mutations in NGLY1as a cause of congenital disorder of deglycosylation: a case presentation
}

\author{
Haixia Ge, Qingbin Wu, Huigang Lu, Yong Huang, Tingting Zhou, Danlin Tan and ZhongqinJin * (D)
}

\begin{abstract}
Background: NGLY1-related congenital disorder of deglycosylation (NGLY1-CDDG) is a multisystemic neurodevelopmental disorder in which affected individuals show developmental delay, epilepsy, intellectual disability, abnormal liver function, and poor growth. This study presents a 10-month-old female infant with elevated liver transaminases, developmental delay, epilepsy (subclinical seizures), and constipation who possesses two compound heterozygous mutations in NGLY1.

Case presentation: The proband was admitted to the Department of Gastroenterology, Children's Hospital of Soochow University, with elevated liver transaminases. She had a history of intrauterine growth retardation and exhibited elevated transaminases, global developmental delay, seizures and light constipation during early infancy. Whole-exome sequencing (WES) and Sanger sequencing revealed two compound heterozygous mutations in NGLY1 that had been inherited in an autosomal recessive manner from her parents. One was a termination mutation, c.1168C > T (p.R390*), and the other was a missense mutation, c.1156G > T (p.D386Y). NGLY1-CDDG is a rare disorder, with a few dozen cases. The two mutations of this proband has not been previously identified.

Conclusions: This study investigated a Chinese proband with NGLY1-CDDG born from healthy parents who was studied using WES and Sanger sequencing to identify the causative mutations. We identified two novel compound heterozygous mutations in NGLY1, c.1168C > T (p.R390*)/c.1156G > T (p.D386Y), which are probably causative of disease.
\end{abstract}

Keywords: NGLY1, Congenital disorder of deglycosylation, Elevated transaminase developmental delay, Seizure, Constipation

\section{Background}

NGLY1-CDDG is a multisystemic neurodevelopmental disorder (Online Mendelian Inheritance in Man: 615273). It is an autosomal recessive disorder with characteristic of mild to profound developmental delay and intellectual disability, elevated liver transaminases, hypoalacrima, and a complex hyperkinetic movement disorder that can include choreiform, athetoid, dystonic, myoclonic, action tremor, and dysmetric movements [1-4].

\footnotetext{
*Correspondence: qingzhongjin@126.com

Department of Gastroenterology, Children's Hospital of Soochow University, Suzhou, Jiangsu, China
}

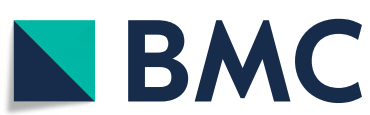

NGLY1-CDDG was first described by Need AC et al. They used whole-exome sequencing to study undiagnosed genetic diseases, and found two loss-of-function mutations in a patient who may have a congenital disorder of glycosylation [5]. NGLY1-CDDG is caused by mutations in NGLY1, which encodes the enzyme $\mathrm{N}$ glycanase 1, which is involved in the deglycosylation of glycoproteins, an essential step in the endoplasmic reticulum-associated degradation (ERAD) pathway [6]. NGLY1-CDDG is a rare disease, and only a few dozen cases have been described. One case was from China [7]. Here, we describe a Chinese infant with elevated liver

(c) The Author(s). 2020 Open Access This article is licensed under a Creative Commons Attribution 4.0 International License, which permits use, sharing, adaptation, distribution and reproduction in any medium or format, as long as you give appropriate credit to the original author(s) and the source, provide a link to the Creative Commons licence, and indicate if changes were made. The images or other third party material in this article are included in the article's Creative Commons licence, unless indicated otherwise in a credit line to the material. If material is not included in the article's Creative Commons licence and your intended use is not permitted by statutory regulation or exceeds the permitted use, you will need to obtain permission directly from the copyright holder. To view a copy of this licence, visit http://creativecommons.org/licenses/by/4.0/ The Creative Commons Public Domain Dedication waiver (http://creativecommons.org/publicdomain/zero/1.0/) applies to the data made available in this article, unless otherwise stated in a credit line to the data. 
transaminases, developmental delay, epilepsy (subclinical seizures) and light constipation who possesses two novel compound heterozygous mutations in NGLY1: a missense mutation and a termination mutation.

\section{Case report}

In 2017, a 10-month-old female infant was admitted to the Department of Gastroenterology, Children's Hospital of Soochow University, because of a history of elevated liver transaminases for more than 3 months. She was born at 30 weeks of gestation, her birth weight was 2.35 $\mathrm{kg}$, and she had a history of intrauterine growth retardation. She had shown global developmental delay since birth. She defecated once every 3-4 days and was crying and restless during defecation. Her parents were physically healthy and were unrelated. She had a brother who died of "convulsion" at the age of 10 months, and there was no family history of inherited diseases.

Physical examination was normal except for slightly high ankle tension. Her liver biochemical profile revealed elevated levels of alanine transaminase ( $147 \mathrm{U} / \mathrm{L}$; normal range, 5-40 U/L) and aspartate transaminase $(112 \mathrm{U} / \mathrm{L}$; normal range, $8-40 \mathrm{U} / \mathrm{L}$ ). Blood tests revealed mildly elevated levels of lactate $(4 \mathrm{mmol} / \mathrm{L}$; normal range, $0.5-$ $2.5 \mathrm{mmol} / \mathrm{L}$ ) and normal levels of IgG, IgA, IgM, and IgE immunoglobulins. Lymphocyte subset analysis was normal, as was blood coagulation function, thyroid function, blood tandem mass spectrometry, and levels of trace elements, ammonia, alpha foetal protein, and urine reducing substances. Pathogen testing was positive for cytomegalovirus IgM, and PCR for cytomegalovirus DNA in peripheral blood revealed the presence of $2.54 \times 10^{3}$ copies $/ \mathrm{ml}$; other pathogens such as EpsteinBarr virus and hepatitis $\mathrm{A}, \mathrm{B}, \mathrm{C}$, and $\mathrm{E}$ were all negative. Ambulatory electroencephalography (EEG) monitoring suggested epilepsy in the form of subclinical seizures. Magnetic resonance imaging of the brain demonstrated increased extracerebral space (Fig. 1). After the patient admission into hospital, she was treated with rehabilitation training and oral compound glycyrrhizin tablets $(2.5 \mathrm{mg} / \mathrm{kg}$ per day) for 10 days. Liver transaminase levels were slightly reduced compared to the first presentation.

\section{Genetic testing and results}

With the consent of the patient's parents, whole exome sequencing (WES) was carried out to screen the mutational sites. Peripheral blood samples from the proband and his parents were collected and sent to Running Gene Inc. (Beijing, China). Following the manufacturer's instructions, DNA samples were extracted using DNA Isolation Kits (Bioteke, Beijing, China; AU1802) and prepared as Illumina sequencing libraries using KAPA Library Preparation Kit (Kapa Biosystems, Beijing, China; KR0453). The libraries were estimated with Qubit dsDNA HS Assay kits (Invitrogen, CA, USA; Q32851). Hybridization of the pooled libraries and removal of non-hybridized DNA fragments were carried out according to the Agilent SureSelectXT2 Target Enrichment System (Agilent, CA, USA). Captured DNA samples were sequenced as paired-end 200-bp reads on the HiSeq2500 platform (Illumina, CA, USA). Functional impacts of the variants were predicted by PolyPhen-2, SIFT, and Mutation Taster prediction software. Variant interpretation was manipulated according to guidelines from the American College of Medical Genetics and Genomics (ACMG) [8]. Sanger sequencing was used to verify genetic mutations which detected by WES. Amplifications were executed with primers targeting the variant loci as follows: forward, $5^{\prime}$-TGAAATGAGACAGT TTAATCCAAAA-3' (chr3:25775467-F-552) and reverse 5'-CAGGAGGCTGAGATACGAGAA-3' (chr3:257754 67-R-552) for c.1168C > T and c.1156G > T in NGLY1.

WES identified two compound heterozygous variants in the proband in exon 8 of NGLY1. c. $1168 \mathrm{C}>\mathrm{T}$ (p.R390*) had been inherited from her mother, and c.1156G > T (p.D386Y) from her father. The mutations were confirmed by Sanger sequencing (Fig. 2). Since the

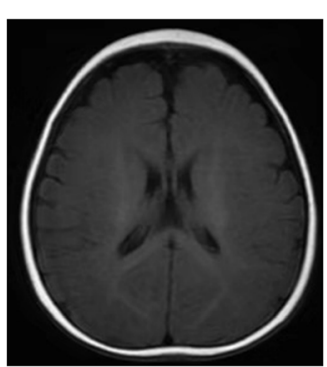

$1 \mathrm{~A}$

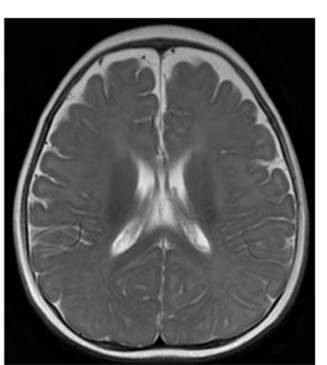

$1 \mathrm{~B}$

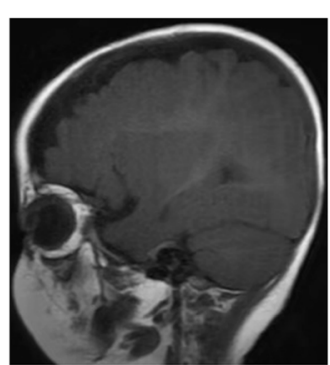

$1 \mathrm{C}$

Fig. 1 Magnetic resonance imaging of the proband's brain. 1A Transverse position (T1WI). An increased distance can be seen between the left frontal lobe and the inner plate of the adjacent skull. 1B Transverse position (T2WI). The increased extracerebral space in the left forehead is filled with cerebrospinal fluid, and the local sulcus is widened. 1C Sagittal position (T1WI). Widening of the extracerebral space is seen in the prefrontal lobe 


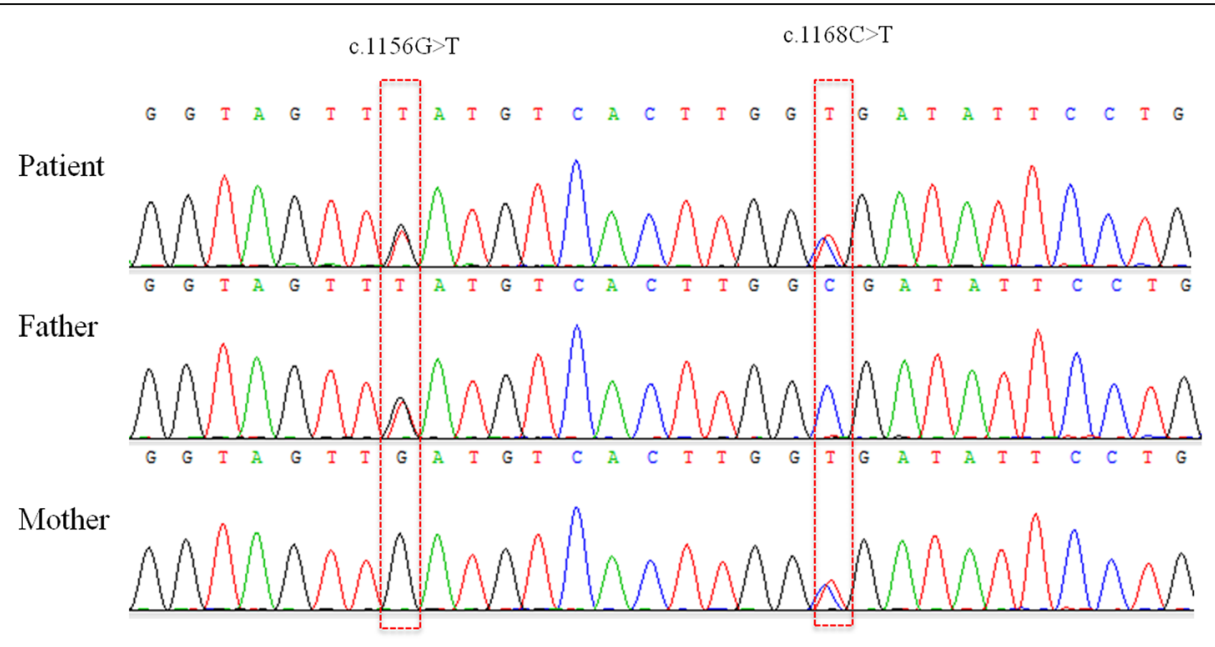

Fig. 2 Electropherograms of NGLY1 exon 8 for the proband and her family. Patient The proband carries compound heterozygous mutations (c.1168C > T [p.R390*] and c.1156G > T [p.D386Y]). Father Her father carries c.1156G > T. Mother Her mother carries c.1168C > T

nonsense mutation c.1168C > T (p.R390*) led to cessation of amino acid translation and was extremely infrequent in GnomAD, ExAC, and 1000 Genomes (PM2). Therefore, c.1168C > T (p.R390") was classified as "pathogenic" according to the ACMG [8]. Moreover, the missense mutation c.1156G > T (p.D386Y) was absent from the 1000 Genomes Project and extremely infrequent in ExAC and GenomeAD (PM2). Additionally, the results of the prediction software were PolyPhen-2 (score 0.91219), SIFT (score 0.97092), and Mutation Taster (score 0.81033) (PP3). Thus, c.1156G > T (p.D386Y) was considered a variant of uncertain significance (VUS). The two compound heterozygous mutations had not previously been described in association with NGLY1-CDDG, and both were highly conserved among a variety of species (Fig. 3 https://genome.ucsc.edu/).

\section{Discussion and conclusions}

NGLY1-CDDG is a rare autosomal recessive hereditary disease, of which a few dozen cases have previously been described $[1-7,9]$. The incidence rate of this disease is unknown. Mutation of NGLY1, which encodescytoplasmic protein $\mathrm{N}$-glycanase 1 , has been demonstrated as a cause of NGLY1-CDDG. In this report, we present a case involving a Chinese female with NGLY1-CDDG who presented with elevated liver transaminases, developmental delay, epilepsy (subclinical seizures) and constipation. WES identified novel compound heterozygousmutations c.1168C $>\mathrm{T} / \mathrm{c} .1156 \mathrm{G}>\mathrm{T}$ in exon 8 of NGLY1 in the patient, which were inherited from her mother and father. Sanger sequencing affirmed these mutations. Termination of the mutation at c.1168C $>\mathrm{T}$ leads to cessation of amino acid translation, and the missense mutation c.1156G $>\mathrm{T}$ causes an amino acid change (aspartic $>$ tyrosine).
NGLY1 is located on chromosome $3 \mathrm{p} 24.2$, which has 12 exons, and encodes the cytoplasmic protein $\mathrm{N}$ glycanase 1(654-amino acid), which plays an important role in the deglycosylation of cytoplasmic N-linked glycoproteins and glycopeptides [10-12]. In 2012 [5], Need et al. used WES to study unidentified genetic diseases and first reported the relationship between NGLY1 mutations and congenital glycosylation disorder. To date, many mutations in NGLY1 have been reported in the Human Gene Mutation Database. The most common pathogenic variant is c.1201A $>\mathrm{T}$, accounting for approximately one-third of pathogenic alleles [4].

$\mathrm{N}$-glycanase 1 functions in the quality control system for newly synthesized glycoproteins in the endoplasmic reticulum, where misfolded glycoproteins are retrotranslocated to the cytosol for degradation, and it also plays a critical role in MHC class I-mediated antigen presentation [13]. Clinical manifestations of NGLY1-deficient patients include abnormal tear production, choreoathetosis, and liver disease, global developmental delay, acquired microcephaly, hypotonia, EEG abnormalities with or without overt seizures, brain imaging abnormalities, peripheral neuropathy, constipation, and a history of intrauterine growth retardation osteopenia, hypocholesterolaemia, difficult swallowing, transient hepatomegaly, anhydrosis, undescended testes, pain insensitivity, low total protein and albumin in the cerebrospinal fluid, and so on $[1,3,4]$.

Formal diagnostic criteria of NGLY1-CDDG have not been established. This disorder can be diagnosed if molecular genetic testing finds biallelic pathogenic variants of NGLY1. No US Food and Drug Association-approved treatments for NGLY1-CDDG currently exist, but enzyme replacement therapy is being evaluated in the preclinical arena [14]. Moreover, preclinical screens for endo-beta-Nacetylglucosaminidase inhibitors are also underway [15]. 


\section{Human DKPLLYEIGWGKKLSYVIAFSKDEVVDVTWRYSCKHEEVIARRTKVKEALLRDTINGLNK \\ Danre DKPLLYEVGWGKKLSYILAFSKDQVADVTWRYSCKHPEVLSRRTQVQETWLLLWILNKL.NA \\ Bovine DKPLLYEVGWGKKLSYILAFSKDQVADVTWRYSCKHPEVLSRRTQVQETWLLHULNKLNA \\ Chicken DKPLLYEIGWGKKLSYVIAFSKDEVVDVTWRYSCKHQEVLTRRTALSEAKLRETINAINK \\ Youse DKPLLYEIGWGKKLSYIIAFSKDEVVDVTWRYSCKHDEVISRRTKVKEELLRETINGLNK \\ Rat DKPLLYEIGWGKKLSYIIAFSKDEVVDVTWRYSCKHEEWMSRRTKVKEELLLRETINGLNK}

Fig. 3 Conservation of the NGLY1 protein sequence among species. The residues mutated in the proband and her family are highlighted

Here, we describe a case of NGLY1-CDDG with novel compound heterozygous mutations in NGLY1. Elevated liver transaminases, developmental delay, constipation, and EEG abnormalities with subclinical seizures were found in this patient, which were consistent with previous reports. Both mutations are predicted to abolish NGLY1 protein, and neither has previously been identified. The patient was treated with oral compound glycyrrhizin tablets, and liver transaminase levels were slightly reduced compared to the first presentation.

In summary, we report a case of a Chinese female with NGLY1 CDDG, which a few dozen cases have previously been described. In this case, we provide new gene mutation sites of NGLY1, and this may offer help for the diagnosis of NGLY1-CDDG in the future.

\section{Abbreviations \\ NGLY1-CDDG: NGLY1-related congenital disorder of deglycosylation; WES: Whole-exome sequencing; ERAD: Endoplasmic reticulum-associated degradation; EEG: Electroencephalography; ACMG: American College of Medical Genetics and Genomics}

\section{Acknowledgements}

The authors thank the family for participating and supporting this study.

\section{Authors' contributions}

ZJ designed the study; HG and ZJ drafted and revised the manuscript; QW, $H L, Y H, T Z$, and DT acquired, analysed, and interpreted the data. All authors read and approved the final manuscript.

\section{Funding}

Not applicable.

\section{Availability of data and materials}

The raw datasets generated and/or analysed during the current study are not publicly available in order to protect participant confidentiality. The data and materials are available from the corresponding author (ZJ) upon reasonable request.

\section{Ethics approval and consent to participate}

Written consent to participate in this study was obtained from parents of the patient. This study was approved by the human ethics committees of the Children's Hospital of Soochow University (the committee's reference number: (2020) No.12)

\section{Consent for publication}

Written consent for publication of this case report was obtained from parents of the patient.
Received: 10 March 2020 Accepted: 10 June 2020

Published online: 23 June 2020

\section{References}

1. Enns GM, Shashi V, Bainbridge M, Gambello MJ, Zahir FR, Bast T, et al. Mutations in NGLY1 cause an inherited disorder of the endoplasmic reticulum-associated degradation pathway. Genet Med. 2014;16:751-8.

2. Heeley J, Shinawi M. Multi-systemic involvement in NGLY1-related disorder caused by two novel mutations. Am J Med Genet A. 2015;167A:816-20.

3. Caglayan AO, Comu S, Baranoski JF, Parman Y, Kaymakcalan H, Akgumus GT, et al. NGLY1 mutation causes neuromotor impairment, intellectual disability, and neuropathy. Eur J Med Genet. 2015;58:39-43.

4. Lam C, Ferreira C, Krasnewich D, Toro C, Latham L, Zein WM, et al. Prospective phenotyping of NGLY1-CDDG, the first congenital disorder of deglycosylation. Genet Med. 2017;19:160-8.

5. Need AC, Shashi V, Hitomi Y, Schoch K, Shianna KV, McDonald MT, et al. Clinical application ofexome sequencing in undiagnosed genetic conditions. J Med Genet. 2012;49(6):353-61.

6. Heeley J, Shinawi M. Multi-systemic involvement in NGLY1-related disorder caused by two novel mutations. Am J Genet A. 2015;167(4):816-20.

7. Zhi LI, Fang LIU. NGLY1 gene mutation causes congenital disorder of glycosylation type IV: a family report. J Clin Pediatr. 2018;36(12):904-6 (Chinese article with english abstract).

8. Kalia SS, Adelman K, Bale SJ, Chung WK, Eng C, Evans JP, et al. Recommendations for reporting of secondary findings in clinical exome and genome sequencing, 2016 update (ACMG SF v2.0): a policy statement of the American College of Medical Genetics and Genomics. Genet Med. 2017;19(2):249-55.

9. Bosch DG, Boonstra FN, de Leeuw N, Pfundt R, Nillesen WM, de Ligt J, et al. Novel genetic causes for cerebral visual impairment. Eur J Hum Genet. 2016; 24:660-5.

10. Suzuki T, Kwofie MA, Lennarz WJ. Ngly1, a mouse gene encoding a deglycosylating enzyme implicated in proteasomal degradation: expression, genomic organization, and chromosomal mapping. Biochem Biophys Res Commun. 2003;304:326-32.

11. Hirsch C, Blom D, Ploegh HL. A role for N-glycanase inthe cytosolic turnover of glycoproteins. EMBO J. 2003;22:1036-46.

12. Suzuki T. Cytoplasmic peptide: N-glycanase and catabolic pathway for free N-glycans in the cytosol. Semin Cell Dev Biol. 2007;18:762-9.

13. Suzuki T, Huang C, Fujihira H. The cytoplasmic peptide: N-glycanase (NGLY1) - structure, expression and cellularfunctions. Gene. 2016;577(1):1-7.

14. Lam C, Wolfe L, Need A, et al. NGLY1-related congenital disorder of deglycosylation. In: Adam MP, Ardinger HH, Pagon RA, et al., editors. GeneReviews ${ }^{\oplus}$ [Internet]. Seattle: University of Washington; 2018. p. 1-17.

15. Bi Y, Might M, Vankayalapati $H$, Kuberan B. Repurposing of proton pump inhibitors as first identified small molecule inhibitors of endo- $\beta-\mathrm{N}$ acetylglucosaminidase (ENGase) for the treatment of NGLY1 deficiency, a rare genetic disease. Bioorg Med Chem Lett. 2017;27:2962-6.

\section{Publisher's Note}

Springer Nature remains neutral with regard to jurisdictional claims in published maps and institutional affiliations. 